Acta Crystallographica Section E

\section{Structure Reports}

Online

ISSN 1600-5368

\section{Desipraminium picrate monohydrate}

\author{
William T. A. Harrison, ${ }^{a}$ M. T. Swamy, ${ }^{b}$ P. Nagaraja, \\ H. S. Yathirajanc and B. Narayana ${ }^{\text {d }}$
}

${ }^{a}$ Department of Chemistry, University of Aberdeen, Meston Walk, Aberdeen AB24 3UE, Scotland, 'b Department of Chemistry, Sambhram Institute of Technology, Bangalore 560 097, India, ' Department of Studies in Chemistry, University of Mysore, Manasagangotri, Mysore 570 006, India, and ${ }^{\mathbf{d}}$ Department of Studies in Chemistry, Mangalore University, Mangalagangotri 574 199, India

Correspondence e-mail: w.harrison@abdn.ac.uk

Received 20 August 2007; accepted 21 August 2007

Key indicators: single-crystal X-ray study; $T=291 \mathrm{~K}$; mean $\sigma(\mathrm{C}-\mathrm{C})=0.004 \AA$; $\mathrm{H}$ atom completeness $93 \%$; disorder in main residue; $R$ factor $=0.067 ; w R$ factor $=$ 0.220 ; data-to-parameter ratio $=14.1$.

In the title compound, $\mathrm{C}_{18} \mathrm{H}_{23} \mathrm{~N}_{2}{ }^{+} \cdot \mathrm{C}_{6} \mathrm{H}_{2} \mathrm{~N}_{3} \mathrm{O}_{7}{ }^{-} \cdot \mathrm{H}_{2} \mathrm{O}$, the cation and anion are linked by a bifurcated $\mathrm{N}-\mathrm{H} \cdots(\mathrm{O}, \mathrm{O})$ hydrogen bond. A second $\mathrm{N}-\mathrm{H} \cdots \mathrm{O}$ interaction connects the cation to the solvent water molecule. The dihedral angle between the aromatic ring planes in the cation is $53.92(8)^{\circ}$. One of the nitro groups of the anion is disordered over two orientations in a ratio of approximately $0.84: 0.16$.

\section{Related literature}

For related structures, see: Portalone et al. (2007); Harrison, Ashok et al. (2007); Harrison, Bindya et al. (2007). For background, see: Cohen et al. (1990); Ahmed et al. (2002).

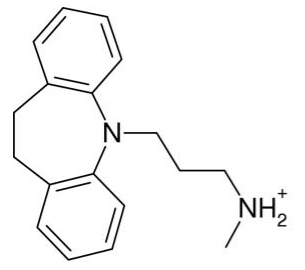<smiles>O=[N+]([O-])c1cc([N+](=O)[O-])c([O-])c([N+](=O)[O-])c1</smiles>

\section{Experimental}

Crystal data

$\mathrm{C}_{18} \mathrm{H}_{23} \mathrm{~N}_{2}{ }^{+} \cdot \mathrm{C}_{6} \mathrm{H}_{2} \mathrm{~N}_{3} \mathrm{O}_{7}{ }^{-} \cdot \mathrm{H}_{2} \mathrm{O}$

$M_{r}=513.51$

Triclinic, $P \overline{1}$

$a=10.3126$ (4) §

$b=11.2594(5) \AA$

$c=11.4304(5) \AA$

$\alpha=72.162(1)^{\circ}$

$\beta=79.288(1)^{\circ}$
Data collection

Bruker SMART1000 CCD

diffractometer

Absorption correction: multi-scan

(SADABS; Bruker, 1999)

$T_{\min }=0.943, T_{\max }=0.954$

Refinement

$R\left[F^{2}>2 \sigma\left(F^{2}\right)\right]=0.067$

$w R\left(F^{2}\right)=0.220$

$S=1.04$

4865 reflections

11334 measured reflections 4865 independent reflections 3688 reflections with $I>2 \sigma(I)$ $R_{\text {int }}=0.015$

\section{Table 1}

Hydrogen-bond geometry $\left(\AA{ }^{\circ}\right)$.

\begin{tabular}{lllll}
\hline$D-\mathrm{H} \cdots A$ & $D-\mathrm{H}$ & $\mathrm{H} \cdots A$ & $D \cdots A$ & $D-\mathrm{H} \cdots A$ \\
\hline $\mathrm{N} 2-\mathrm{H} 1 \mathrm{~N} \cdots \mathrm{O} 11$ & 0.86 & 2.06 & $2.817(3)$ & 146 \\
$\mathrm{~N} 2-\mathrm{H} 1 \mathrm{~N} \cdots \mathrm{O} 12$ & 0.86 & 2.38 & $3.001(3)$ & 129 \\
$\mathrm{~N} 2-\mathrm{H} 2 \mathrm{~N} \cdots \mathrm{O} 21$ & 0.86 & 2.03 & $2.873(4)$ & 170 \\
\hline
\end{tabular}

Data collection: SMART (Bruker, 1999); cell refinement: SAINT (Bruker, 1999); data reduction: $S A I N T$; program(s) used to solve structure: SHELXS97 (Sheldrick, 1997); program(s) used to refine structure: SHELXL97 (Sheldrick, 1997); molecular graphics: ORTEP-3 (Farrugia, 1997); software used to prepare material for publication: SHELXL97.

MTS thanks Sambhram Institute of Technology for research facilities.

Supplementary data and figures for this paper are available from the IUCr electronic archives (Reference: BT2487).

\section{References}

Ahmed, S. A., Silwadi, M. F. \& Khatoon, B. A. (2002). J. Pharm. Biomed. Anal. 28, 501-507.

Bruker (1999). SMART, SAINT and $S A D A B S$. Bruker AXS Inc., Madison, Wisconsin, USA.

Cohen, M. D., Finberg, J., Dibner-Dunlap, M., Yuih, S. N. \& Thames, M. D. (1990). Am. J. Physiol. Regul. Integr. Comp. Physiol. 258, 876-882.

Farrugia, L. J. (1997). J. Appl. Cryst. 30, 565.

Harrison, W. T. A., Ashok, M. A., Yathirajan, H. S. \& Narayana Achar, B. (2007). Acta Cryst. E63, o3277.

Harrison, W. T. A., Bindya, S., Ashok, M. A., Yathirajan, H. S. \& Narayana, B. (2007). Acta Cryst. E63, o3143.

Portalone, G., Colapietro, M., Bindya, S., Ashok, M. A. \& Yathirajan, H. S. (2007). Acta Cryst. E63, o746-0747.

Sheldrick, G. M. (1997). SHELXS97 and SHELXL97. University of Göttingen, Germany. 


\section{supplementary materials}


Acta Cryst. (2007). E63, o3892 [ doi:10.1107/S1600536807041219]

\title{
Desipraminium picrate monohydrate
}

\author{
W. T. A. Harrison, M. T. Swamy, P. Nagaraja, H. S. Yathirajan and B. Narayana
}

\section{Comment}

Desipramine, 10,11-dihydro-5-[3-(methylamino)propyl]-5H-dibenz[b,f]azepine, $\mathrm{C}_{18} \mathrm{H}_{22} \mathrm{~N}_{2}$, is a tricyclic antidepressant (TCA) and pain reliever that inhibits the reuptake of norepinephrine. Medical applications (Cohen et al., 1990) usually utilize desipramine as its hydrochloride salt, i.e. $\mathrm{C}_{18} \mathrm{H}_{23} \mathrm{~N}_{2}{ }^{+} \cdot \mathrm{Cl}^{-}$. A recent proposed new application of desipramine hydrochloride as a reagent for the detection of microamounts of blood in urine (Ahmed et al., 2002).

In continuation of our work on the picrate salts of pharmaceutical compounds (Portalone et al., 2007; Harrison, Ashok et al., 2007; Harrison, Bindya et al., 2007), we now report the structure of the title compound, (I).

The structure of (I) (Fig. 1) shows that proton transfer from picric acid (pa) to desipramine (dp) has occurred, and that the secondary-amine $\mathrm{N}$ atom of the side chain has been protonated to yield an $-\mathrm{NH}_{2}{ }^{+}$- grouping. The dihedral angle between the $\mathrm{C} 1-\mathrm{C} 6$ and $\mathrm{C} 9-\mathrm{C} 14$ ring planes in (I) is $53.92(8)^{\circ}$. The bond-angle sum for $\mathrm{N} 1$ is $360.0^{\circ}$, indicating $s p^{2}$ hybridization. However, the unobserved $\mathrm{p}$ orbital appears not to be well aligned to overlap with the $\pi$ clouds of the adjacent benzene rings, as the $\mathrm{C} 1 / \mathrm{C} 14 / \mathrm{C} 15 / \mathrm{N} 1$ grouping makes dihedral angles of $65.28(10)^{\circ}$ and $44.78(11)^{\circ}$ with the $\mathrm{C} 1-\mathrm{C} 6$ and $\mathrm{C} 9-\mathrm{C} 14$ ring planes, respectively. The seven-membered ring in (I) approximates to a boat, with $\mathrm{C} 1 / \mathrm{C} 6 / \mathrm{C} 8 / \mathrm{C} 9$ almost co-planar (r.m.s. deviation $=0.002 \AA$ ), $\mathrm{C} 7$ forming the prow [deviation $=0.721$ (3) $\AA$ ] and N1 and C14 the stern [deviations $=0.787$ (4) and 0.351 (4) $\AA$, respectively].

The significant variation of the $\mathrm{C}-\mathrm{C}$ bond lengths around the picrate aromatic ring in (I) are normal (Harrison, Ashok et al., 2007). The N13 nitro group is disordered over two orientations.

In the crystal, the cation and anion are linked by a bifurcated $\mathrm{N}-\mathrm{H} \cdots(\mathrm{O}, O)$ hydrogen bond (Table 1), as also seen in related compounds (Harrison, Ashok et al., 2007). The cation also bonds to the $\mathrm{O} 21$ water molecule with an $\mathrm{N}-\mathrm{H} \cdots \mathrm{O}$ link. Unfortunately, the water molecule $\mathrm{H}$ atoms were not located in this study. One of the $\mathrm{H}$ atoms is probably involved in an $\mathrm{H}$ bond to $\mathrm{O} 16[\mathrm{O} 21 \cdots \mathrm{O} 16=2.667(5) \AA]$, but there is no obvious second $\mathrm{H}$ bond and geometrical placement of the $\mathrm{H}$ atoms was not attempted.

\section{Experimental}

Desipramine hydrochloride $(0.8 \mathrm{~g}, 0.05 \mathrm{~mol})$ and picric acid $(0.6 \mathrm{~g}, 0.05 \mathrm{~mol})$ were dissolved in distilled water $(50 \mathrm{ml})$, mixed and stirred well in a beaker at room temperature. The separated bright yellow salt was washed well with distilled water, filtered and dried in a vacuum desiccator over $\mathrm{P}_{2} \mathrm{O}_{5}$. Yellow blocks of (I) were recrystallized from absolute ethanol (m.p.: $387 \mathrm{~K}$ ). The water of crystallization in (I) was presumably incorporated from the atmosphere. 


\section{supplementary materials}

\section{Refinement}

One of the nitro groups of the anion is disordered over two orientations in a 0.840 (6):0.160 (6) ratio. The N-bound $\mathrm{H}$ atoms were located in a differenc map, then relocated to idealized locations $(\mathrm{N}-\mathrm{H}=0.86 \AA)$ and refined as riding with $U_{\text {iso }}(\mathrm{H})$ $=1.2 U_{\text {eq }}(\mathrm{N})$. The C-bound $\mathrm{H}$ atoms were geometrically placed $(\mathrm{C}-\mathrm{H}=0.93-0.96 \AA)$ and refined as riding with $U_{\text {iso }}(\mathrm{H})=$ $1.2 U_{\mathrm{eq}}(\mathrm{C})$ or $1.5 U_{\mathrm{eq}}$ (methyl $\mathrm{C}$ ). The methyl group was allowed to rotate, but not to tip, to best fit the electron density. The $\mathrm{H}$ atoms of the water molecule could not be located in the present study.

\section{Figures}

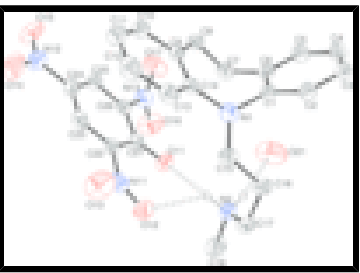

Fig. 1. View of the molecular structure of (I) showing 30\% displacement ellipsoids (arbitrary spheres for the $\mathrm{H}$ atoms). The hydrogen bonds are shown as double-dashed lines. Only the $\mathrm{N}$ bound $\mathrm{H}$ atoms and the major disorder component for the N13 nitro group are shown.

\section{Desipraminium picrate monohydrate}

\section{Crystal data}

$\mathrm{C}_{18} \mathrm{H}_{23} \mathrm{~N}_{2}^{+} \cdot \mathrm{C}_{6} \mathrm{H}_{2} \mathrm{~N}_{3} \mathrm{O}_{7}^{-} \cdot \mathrm{H}_{2} \mathrm{O}$

$M_{r}=513.51$

Triclinic, $P \overline{1}$

Hall symbol: -P 1

$a=10.3126(4) \AA$

$b=11.2594(5) \AA$

$c=11.4304(5) \AA$

$\alpha=72.162(1)^{\circ}$

$\beta=79.288(1)^{\circ}$

$\gamma=87.233(1)^{\circ}$

$V=1241.35(9) \AA^{3}$

\section{Data collection}

\section{Bruker SMART1000 CCD}

diffractometer

Radiation source: fine-focus sealed tube

Monochromator: graphite

$T=291(2) \mathrm{K}$

$\omega$ scans

Absorption correction: multi-scan

(SADABS; Bruker, 1999)

$T_{\min }=0.943, T_{\max }=0.954$

$$
\begin{aligned}
& Z=2 \\
& F_{000}=540 \\
& D_{\mathrm{x}}=1.374 \mathrm{Mg} \mathrm{m}^{-3} \\
& \text { Mo } K \alpha \text { radiation } \\
& \lambda=0.71073 \AA \\
& \text { Cell parameters from } 5773 \text { reflections } \\
& \theta=2.3-27.5^{\circ} \\
& \mu=0.11 \mathrm{~mm}^{-1} \\
& T=291(2) \mathrm{K} \\
& \text { Chunk, yellow } \\
& 0.60 \times 0.50 \times 0.45 \mathrm{~mm}
\end{aligned}
$$

4865 independent reflections

3688 reflections with $I>2 \sigma(I)$

$R_{\text {int }}=0.015$

$\theta_{\text {max }}=26.0^{\circ}$

$\theta_{\min }=2.3^{\circ}$

$h=-11 \rightarrow 12$

$k=-13 \rightarrow 13$ 
11334 measured reflections

\section{Refinement}

Refinement on $F^{2}$

Least-squares matrix: full

$R\left[F^{2}>2 \sigma\left(F^{2}\right)\right]=0.067$

$w R\left(F^{2}\right)=0.220$

$S=1.04$

4865 reflections

344 parameters

Primary atom site location: structure-invariant direct methods

$$
l=-14 \rightarrow 14
$$

Secondary atom site location: difference Fourier map

Hydrogen site location: difference Fourier map

H-atom parameters constrained

$$
\begin{aligned}
& w=1 /\left[\sigma^{2}\left(F_{\mathrm{o}}{ }^{2}\right)+(0.1277 P)^{2}+0.5257 P\right] \\
& \text { where } P=\left(F_{\mathrm{o}}{ }^{2}+2 F_{\mathrm{c}}{ }^{2}\right) / 3 \\
& (\Delta / \sigma)_{\max }<0.001 \\
& \Delta \rho_{\max }=0.47 \text { e } \AA^{-3} \\
& \Delta \rho_{\min }=-0.58 \text { e } \AA^{-3}
\end{aligned}
$$

Extinction correction: none

\section{Special details}

Geometry. All e.s.d.'s (except the e.s.d. in the dihedral angle between two 1.s. planes) are estimated using the full covariance matrix. The cell e.s.d.'s are taken into account individually in the estimation of e.s.d.'s in distances, angles and torsion angles; correlations between e.s.d.'s in cell parameters are only used when they are defined by crystal symmetry. An approximate (isotropic) treatment of cell e.s.d.'s is used for estimating e.s.d.'s involving 1.s. planes.

\begin{tabular}{|c|c|c|c|c|}
\hline & $x$ & $y$ & $z$ & $U_{\text {iso }} * / U_{\text {eq }}$ \\
\hline $\mathrm{C} 1$ & $0.3705(2)$ & 0.18454 (19) & $0.4622(2)$ & $0.0422(5)$ \\
\hline $\mathrm{C} 2$ & $0.4803(3)$ & $0.1592(2)$ & $0.5201(3)$ & $0.0557(6)$ \\
\hline $\mathrm{H} 2$ & 0.5632 & 0.1876 & 0.4743 & $0.067^{*}$ \\
\hline $\mathrm{C} 3$ & $0.4669(3)$ & 0.0915 (3) & $0.6462(3)$ & $0.0676(8)$ \\
\hline H3 & 0.5407 & 0.0748 & 0.6844 & $0.081 *$ \\
\hline $\mathrm{C} 4$ & $0.3441(4)$ & $0.0493(3)$ & $0.7141(3)$ & $0.0735(9)$ \\
\hline H4 & 0.3349 & 0.0041 & 0.7982 & $0.088^{*}$ \\
\hline C5 & $0.2351(3)$ & $0.0740(3)$ & $0.6576(2)$ & $0.0614(7)$ \\
\hline H5 & 0.1525 & 0.0456 & 0.7043 & $0.074 *$ \\
\hline C6 & $0.2464(2)$ & $0.1412(2)$ & $0.5309(2)$ & $0.0447(5)$ \\
\hline $\mathrm{C} 7$ & $0.1308(2)$ & $0.1630(2)$ & $0.4653(2)$ & $0.0499(6)$ \\
\hline $\mathrm{H} 7 \mathrm{~A}$ & 0.1287 & 0.2503 & 0.4171 & $0.060^{*}$ \\
\hline H7B & 0.0499 & 0.1436 & 0.5266 & $0.060 *$ \\
\hline $\mathrm{C} 8$ & $0.1382(2)$ & $0.0830(2)$ & $0.3790(2)$ & $0.0515(6)$ \\
\hline H8A & 0.1503 & -0.0027 & 0.4274 & $0.062 *$ \\
\hline H8B & 0.0533 & 0.0870 & 0.3531 & $0.062 *$ \\
\hline C9 & $0.2437(2)$ & 0.11361 (19) & $0.2627(2)$ & $0.0461(5)$ \\
\hline $\mathrm{C} 10$ & $0.2280(3)$ & $0.0574(2)$ & $0.1725(3)$ & $0.0613(7)$ \\
\hline
\end{tabular}

Refinement. Refinement of $\mathrm{F}^{2}$ against ALL reflections. The weighted $R$-factor $w R$ and goodness of fit $\mathrm{S}$ are based on $\mathrm{F}^{2}$, conventional $R$-factors $R$ are based on $\mathrm{F}$, with $\mathrm{F}$ set to zero for negative $\mathrm{F}^{2}$. The threshold expression of $\mathrm{F}^{2}>2$ sigma $\left(\mathrm{F}^{2}\right)$ is used only for calculating $R$-factors (gt) etc. and is not relevant to the choice of reflections for refinement. $R$-factors based on $\mathrm{F}^{2}$ are statistically about twice as large as those based on F, and R- factors based on ALL data will be even larger.

Fractional atomic coordinates and isotropic or equivalent isotropic displacement parameters $\left(\AA^{2}\right)$ 
supplementary materials

\begin{tabular}{|c|c|c|c|c|c|}
\hline H10 & 0.1550 & 0.0060 & 0.1876 & $0.074 *$ & \\
\hline $\mathrm{C} 11$ & $0.3157(4)$ & $0.0751(3)$ & $0.0634(3)$ & $0.0779(9)$ & \\
\hline H11 & 0.3026 & 0.0356 & 0.0059 & $0.093 *$ & \\
\hline $\mathrm{C} 12$ & $0.4236(4)$ & $0.1518(3)$ & $0.0391(3)$ & $0.0789(10)$ & \\
\hline H12 & 0.4837 & 0.1651 & -0.0354 & $0.095^{*}$ & \\
\hline $\mathrm{C} 13$ & $0.4424(3)$ & $0.2094(2)$ & $0.1266(2)$ & $0.0606(7)$ & \\
\hline H13 & 0.5158 & 0.2608 & 0.1098 & $0.073^{*}$ & \\
\hline $\mathrm{C} 14$ & $0.3542(2)$ & 0.19202 (19) & $0.2385(2)$ & $0.0436(5)$ & \\
\hline $\mathrm{C} 15$ & $0.4229(2)$ & $0.3765(2)$ & $0.2848(2)$ & $0.0475(5)$ & \\
\hline $\mathrm{H} 15 \mathrm{~A}$ & 0.3560 & 0.4218 & 0.2395 & $0.057 *$ & \\
\hline H15B & 0.5017 & 0.3791 & 0.2226 & $0.057 *$ & \\
\hline $\mathrm{C} 16$ & $0.4542(3)$ & $0.4549(2)$ & $0.3614(3)$ & $0.0588(7)$ & \\
\hline H16A & 0.5492 & 0.4615 & 0.3522 & $0.071^{*}$ & \\
\hline H16B & 0.4193 & 0.4139 & 0.4488 & $0.071^{*}$ & \\
\hline $\mathrm{C} 17$ & 0.3969 (3) & $0.5852(2)$ & $0.3232(3)$ & $0.0618(7)$ & \\
\hline H17A & 0.4266 & 0.6343 & 0.3698 & $0.074 *$ & \\
\hline H17B & 0.4294 & 0.6253 & 0.2351 & $0.074^{*}$ & \\
\hline $\mathrm{C} 18$ & $0.1933(4)$ & $0.7103(3)$ & $0.3209(4)$ & $0.0896(11)$ & \\
\hline $\mathrm{H} 18 \mathrm{~A}$ & 0.0988 & 0.7040 & 0.3386 & $0.134 *$ & \\
\hline H18B & 0.2221 & 0.7567 & 0.2348 & $0.134 *$ & \\
\hline $\mathrm{H} 18 \mathrm{C}$ & 0.2221 & 0.7523 & 0.3732 & $0.134 *$ & \\
\hline N1 & $0.3828(2)$ & $0.2574(2)$ & $0.3275(2)$ & $0.0554(5)$ & \\
\hline $\mathrm{N} 2$ & $0.2508(2)$ & $0.5830(2)$ & $0.3460(2)$ & $0.0559(5)$ & \\
\hline $\mathrm{H} 1 \mathrm{~N}$ & 0.2230 & 0.5440 & 0.3014 & $0.067^{*}$ & \\
\hline $\mathrm{H} 2 \mathrm{~N}$ & 0.2161 & 0.5385 & 0.4198 & $0.067 *$ & \\
\hline $\mathrm{C} 21$ & $0.0489(2)$ & $0.4204(2)$ & $0.1836(2)$ & $0.0428(5)$ & \\
\hline $\mathrm{C} 22$ & $0.1382(2)$ & $0.4268(2)$ & $0.0688(2)$ & $0.0442(5)$ & \\
\hline $\mathrm{C} 23$ & $0.1227(3)$ & $0.3566(2)$ & $-0.0082(2)$ & $0.0529(6)$ & \\
\hline $\mathrm{H} 23$ & 0.1833 & 0.3644 & -0.0815 & $0.063^{*}$ & \\
\hline $\mathrm{C} 24$ & $0.0174(3)$ & $0.2750(2)$ & $0.0241(2)$ & $0.0551(6)$ & \\
\hline $\mathrm{C} 25$ & $-0.0728(3)$ & $0.2612(2)$ & $0.1328(2)$ & $0.0541(6)$ & \\
\hline $\mathrm{H} 25$ & -0.1427 & 0.2048 & 0.1550 & $0.065^{*}$ & \\
\hline $\mathrm{C} 26$ & $-0.0576(2)$ & $0.3315(2)$ & $0.2070(2)$ & $0.0462(5)$ & \\
\hline N11 & $0.2504(2)$ & $0.5128(2)$ & $0.0260(2)$ & $0.0598(6)$ & \\
\hline N12 & $-0.0004(3)$ & $0.2036(3)$ & -0.0585 & $0.0804(8)$ & \\
\hline N13 & $-0.1562(2)$ & $0.3130(2)$ & $0.3202(2)$ & $0.0631(6)$ & \\
\hline O11 & 0.05959 (19) & $0.47933(17)$ & $0.25813(17)$ & $0.0595(5)$ & \\
\hline $\mathrm{O} 12$ & $0.2615(2)$ & $0.5890(2)$ & $0.0801(2)$ & $0.0795(6)$ & \\
\hline $\mathrm{O} 13$ & $0.3289(3)$ & $0.5073(3)$ & -0.0639 & $0.1196(11)$ & \\
\hline O14 & $0.0784(4)$ & $0.2197(3)$ & -0.1557 (3) & $0.1102(10)$ & \\
\hline $\mathrm{O} 15$ & -0.0951 & $0.1312(3)$ & -0.0257 (3) & $0.1176(11)$ & \\
\hline O16 & -0.2029 (3) & $0.4031(3)$ & $0.3489(3)$ & 0.1007 (13) & $0.840(6)$ \\
\hline $\mathrm{O} 17$ & -0.1854 (3) & $0.2071(3)$ & $0.3862(3)$ & $0.0971(13)$ & $0.840(6)$ \\
\hline O18 & $-0.1390(18)$ & $0.2968(17)$ & 0.4109 (18) & $0.092(6)^{*}$ & $0.160(6)$ \\
\hline O19 & $-0.278(3)$ & $0.301(2)$ & $0.301(2)$ & $0.144(10)^{*}$ & $0.160(6)$ \\
\hline $\mathrm{O} 21$ & 0.1305 (4) & $0.4099(3)$ & $0.5790(3)$ & $0.1427(15)$ & \\
\hline
\end{tabular}


Atomic displacement parameters $\left(\AA^{2}\right)$

\begin{tabular}{|c|c|c|c|c|c|c|}
\hline & $U^{11}$ & $U^{22}$ & $U^{33}$ & $U^{12}$ & $U^{13}$ & $U^{23}$ \\
\hline $\mathrm{C} 1$ & $0.0435(12)$ & $0.0359(10)$ & $0.0494(12)$ & $0.0018(8)$ & $-0.0119(9)$ & $-0.0144(9)$ \\
\hline $\mathrm{C} 2$ & $0.0515(14)$ & $0.0504(13)$ & $0.0714(16)$ & $0.0057(10)$ & $-0.0231(12)$ & $-0.0210(12)$ \\
\hline $\mathrm{C} 3$ & $0.087(2)$ & $0.0567(15)$ & $0.0725(18)$ & $0.0144(14)$ & $-0.0477(17)$ & $-0.0217(13)$ \\
\hline $\mathrm{C} 4$ & $0.111(3)$ & $0.0626(17)$ & $0.0477(14)$ & $0.0047(17)$ & $-0.0259(16)$ & $-0.0120(12)$ \\
\hline $\mathrm{C} 5$ & $0.0774(18)$ & $0.0564(15)$ & $0.0467(13)$ & $-0.0057(13)$ & $-0.0035(12)$ & -0.0138 \\
\hline C6 & $0.0486(12)$ & $0.0391(11)$ & $0.0457(11)$ & $-0.0002(9)$ & $-0.0054(9)$ & $-0.0136(9)$ \\
\hline $\mathrm{C} 7$ & $0.0376(11)$ & $0.0487(12)$ & $0.0564(13)$ & $-0.0044(9)$ & $-0.0003(10)$ & $-0.0099(10)$ \\
\hline $\mathrm{C} 8$ & $0.0492(13)$ & $0.0418(12)$ & $0.0627(14)$ & $-0.0085(10)$ & $-0.0182(11)$ & $-0.0086(10)$ \\
\hline C9 & $0.0554(13)$ & $0.0331(10)$ & $0.0525(12)$ & 0.0067 (9) & $-0.0223(10)$ & $-0.0105(9)$ \\
\hline $\mathrm{C} 10$ & $0.0791(19)$ & $0.0462(13)$ & $0.0680(16)$ & $0.0080(12)$ & $-0.0317(14)$ & $-0.0214(12)$ \\
\hline $\mathrm{C} 11$ & $0.115(3)$ & $0.0649(18)$ & $0.0684(18)$ & $0.0157(18)$ & $-0.0309(18)$ & $-0.0348(15)$ \\
\hline $\mathrm{C} 12$ & $0.106(3)$ & $0.077(2)$ & $0.0487(15)$ & $0.0230(19)$ & $-0.0022(15)$ & $-0.0218(14)$ \\
\hline $\mathrm{C} 13$ & $0.0653(17)$ & $0.0540(14)$ & $0.0549(14)$ & $0.0046(12)$ & $0.0005(12)$ & $-0.0129(11)$ \\
\hline C14 & $0.0473(12)$ & $0.0356(10)$ & $0.0456(11)$ & $0.0053(9)$ & $-0.0098(9)$ & $-0.0089(9)$ \\
\hline $\mathrm{C} 15$ & $0.0358(11)$ & $0.0431(12)$ & $0.0589(13)$ & $-0.0047(9)$ & $-0.0044(9)$ & $-0.0105(10)$ \\
\hline $\mathrm{C} 16$ & $0.0502(14)$ & $0.0498(13)$ & $0.0802(17)$ & $-0.0091(11)$ & $-0.0226(12)$ & $-0.0174(12)$ \\
\hline $\mathrm{C} 17$ & $0.0684(17)$ & $0.0453(13)$ & $0.0765(17)$ & $-0.0121(12)$ & $-0.0238(14)$ & $-0.0172(12)$ \\
\hline $\mathrm{C} 18$ & 0.111 & $0.071(2)$ & 0.111 & 0.0295 (19) & $-0.051(2)$ & $-0.050(2)$ \\
\hline N1 & $0.0438(11)$ & $0.0580(12)$ & $0.0614(12)$ & 0.0002 (9) & -0.0078 (9) & $-0.0148(10)$ \\
\hline $\mathrm{N} 2$ & $0.0644(13)$ & $0.0542(12)$ & $0.0569(12)$ & $0.0019(10)$ & $-0.0198(10)$ & $-0.0230(9)$ \\
\hline $\mathrm{C} 21$ & $0.0434(12)$ & $0.0413(11)$ & $0.0434(11)$ & 0.0041 (9) & $-0.0129(9)$ & $-0.0100(9)$ \\
\hline $\mathrm{C} 22$ & $0.0386(11)$ & $0.0465(12)$ & 0.0439 & 0.0008 (9) & -0.0108 (9) & $-0.0065(9)$ \\
\hline $\mathrm{C} 23$ & $0.0573(14)$ & $0.0571(14)$ & $0.0418(11)$ & $0.0160(11)$ & $-0.0119(10)$ & $-0.0118(10)$ \\
\hline $\mathrm{C} 24$ & $0.0691(16)$ & $0.0481(13)$ & $0.0561(14)$ & $0.0089(11)$ & $-0.0261(12)$ & $-0.0201(11)$ \\
\hline $\mathrm{C} 25$ & $0.0542(14)$ & $0.0453(13)$ & $0.0645(15)$ & $-0.0034(10)$ & $-0.0236(12)$ & $-0.0108(11)$ \\
\hline $\mathrm{C} 26$ & $0.0408(12)$ & $0.0470(12)$ & $0.0470(12)$ & $0.0020(9)$ & $-0.0094(9)$ & $-0.0082(9)$ \\
\hline N11 & $0.0488(12)$ & $0.0693(14)$ & $0.0516(12)$ & $-0.0075(10)$ & $-0.0080(10)$ & $-0.0036(10)$ \\
\hline $\mathrm{N} 12$ & 0.109 (2) & $0.0686(16)$ & $0.0815(18)$ & $0.0172(16)$ & $-0.0413(17)$ & -0.0378 \\
\hline N13 & $0.0460(12)$ & $0.0755(17)$ & $0.0641(14)$ & $-0.0049(11)$ & $-0.0061(10)$ & $-0.0173(12)$ \\
\hline O11 & $0.0621(11)$ & $0.0657(11)$ & $0.0569(10)$ & $-0.0069(9)$ & $-0.0078(8)$ & $-0.0283(9)$ \\
\hline $\mathrm{O} 12$ & $0.0817(15)$ & $0.0814(14)$ & $0.0741(13)$ & $-0.0331(11)$ & $-0.0150(11)$ & $-0.0161(11)$ \\
\hline $\mathrm{O} 13$ & 0.0870 & $0.154(3)$ & $0.106(2)$ & $-0.0507(18)$ & $0.0476(16)$ & $-0.0526(19)$ \\
\hline $\mathrm{O} 14$ & 0.153 & $0.118(2)$ & $0.0835(17)$ & $0.0271(19)$ & $-0.0289(18)$ & $-0.0645(16)$ \\
\hline $\mathrm{O} 15$ & 0.154 & $0.096(2)$ & 0.134 & $-0.0217(19)$ & $-0.050(2)$ & $-0.0633(18)$ \\
\hline O16 & $0.087(2)$ & $0.103(2)$ & $0.111(2)$ & $-0.0076(17)$ & $0.0254(18)$ & -0.055 (2) \\
\hline $\mathrm{O} 17$ & 0.081 & $0.089(2)$ & $0.087(2)$ & $-0.0174(15)$ & $0.0176(15)$ & $0.0065(16)$ \\
\hline $\mathrm{O} 21$ & 0.215 & $0.099(2)$ & 0.0909 (19) & -0.009 (2) & 0.043 & -0.0353 \\
\hline
\end{tabular}

Geometric parameters $\left(\AA,{ }^{\circ}\right)$

$\begin{array}{ll}\mathrm{C} 1-\mathrm{C} 2 & 1.390(3) \\ \mathrm{C} 1-\mathrm{C} 6 & 1.394(3) \\ \mathrm{C} 1-\mathrm{N} 1 & 1.491(3) \\ \mathrm{C} 2-\mathrm{C} 3 & 1.393(4) \\ \mathrm{C} 2-\mathrm{H} 2 & 0.9300\end{array}$

$$
\begin{aligned}
& \text { C16-C17 } \\
& \text { C16-H16A } \\
& \text { C16-H16B } \\
& \text { C17-N2 } \\
& \text { C17-H17A }
\end{aligned}
$$

$1.521(4)$ 0.9700 0.9700 $1.481(4)$ 0.9700 


\begin{tabular}{|c|c|c|c|}
\hline $\mathrm{C} 3-\mathrm{C} 4$ & $1.378(5)$ & $\mathrm{C} 17-\mathrm{H} 17 \mathrm{~B}$ & 0.9700 \\
\hline $\mathrm{C} 3-\mathrm{H} 3$ & 0.9300 & $\mathrm{C} 18-\mathrm{N} 2$ & $1.489(4)$ \\
\hline $\mathrm{C} 4-\mathrm{C} 5$ & $1.373(4)$ & $\mathrm{C} 18-\mathrm{H} 18 \mathrm{~A}$ & 0.9600 \\
\hline $\mathrm{C} 4-\mathrm{H} 4$ & 0.9300 & $\mathrm{C} 18-\mathrm{H} 18 \mathrm{~B}$ & 0.9600 \\
\hline $\mathrm{C} 5-\mathrm{C} 6$ & $1.399(3)$ & $\mathrm{C} 18-\mathrm{H} 18 \mathrm{C}$ & 0.9600 \\
\hline $\mathrm{C} 5-\mathrm{H} 5$ & 0.9300 & $\mathrm{~N} 2-\mathrm{H} 1 \mathrm{~N}$ & 0.8596 \\
\hline $\mathrm{C} 6-\mathrm{C} 7$ & $1.495(3)$ & $\mathrm{N} 2-\mathrm{H} 2 \mathrm{~N}$ & 0.8577 \\
\hline $\mathrm{C} 7-\mathrm{C} 8$ & $1.516(4)$ & $\mathrm{C} 21-\mathrm{O} 11$ & $1.252(3)$ \\
\hline $\mathrm{C} 7-\mathrm{H} 7 \mathrm{~A}$ & 0.9700 & $\mathrm{C} 21-\mathrm{C} 22$ & $1.440(3)$ \\
\hline C7-H7B & 0.9700 & $\mathrm{C} 21-\mathrm{C} 26$ & $1.453(3)$ \\
\hline $\mathrm{C} 8-\mathrm{C} 9$ & $1.513(4)$ & $\mathrm{C} 22-\mathrm{C} 23$ & $1.385(3)$ \\
\hline $\mathrm{C} 8-\mathrm{H} 8 \mathrm{~A}$ & 0.9700 & $\mathrm{C} 22-\mathrm{N} 11$ & $1.457(3)$ \\
\hline $\mathrm{C} 8-\mathrm{H} 8 \mathrm{~B}$ & 0.9700 & $\mathrm{C} 23-\mathrm{C} 24$ & $1.379(4)$ \\
\hline $\mathrm{C} 9-\mathrm{C} 10$ & $1.401(3)$ & $\mathrm{C} 23-\mathrm{H} 23$ & 0.9300 \\
\hline $\mathrm{C} 9-\mathrm{C} 14$ & $1.409(3)$ & $\mathrm{C} 24-\mathrm{C} 25$ & $1.379(4)$ \\
\hline $\mathrm{C} 10-\mathrm{C} 11$ & $1.363(5)$ & $\mathrm{C} 24-\mathrm{N} 12$ & $1.453(3)$ \\
\hline $\mathrm{C} 10-\mathrm{H} 10$ & 0.9300 & $\mathrm{C} 25-\mathrm{C} 26$ & $1.357(3)$ \\
\hline $\mathrm{C} 11-\mathrm{C} 12$ & $1.376(5)$ & $\mathrm{C} 25-\mathrm{H} 25$ & 0.9300 \\
\hline $\mathrm{C} 11-\mathrm{H} 11$ & 0.9300 & $\mathrm{C} 26-\mathrm{N} 13$ & $1.456(3)$ \\
\hline $\mathrm{C} 12-\mathrm{C} 13$ & $1.394(4)$ & $\mathrm{N} 11-\mathrm{O} 13$ & $1.200(3)$ \\
\hline $\mathrm{C} 12-\mathrm{H} 12$ & 0.9300 & $\mathrm{~N} 11-\mathrm{O} 12$ & $1.222(3)$ \\
\hline $\mathrm{C} 13-\mathrm{C} 14$ & $1.389(3)$ & $\mathrm{N} 12-\mathrm{O} 14$ & $1.218(4)$ \\
\hline $\mathrm{C} 13-\mathrm{H} 13$ & 0.9300 & $\mathrm{~N} 12-\mathrm{O} 15$ & $1.229(4)$ \\
\hline $\mathrm{C} 14-\mathrm{N} 1$ & $1.502(3)$ & $\mathrm{N} 13-\mathrm{O} 18$ & $1.044(19)$ \\
\hline $\mathrm{C} 15-\mathrm{N} 1$ & $1.336(3)$ & $\mathrm{N} 13-\mathrm{O} 16$ & $1.211(4)$ \\
\hline $\mathrm{C} 15-\mathrm{C} 16$ & $1.502(3)$ & $\mathrm{N} 13-\mathrm{O} 17$ & $1.217(4)$ \\
\hline $\mathrm{C} 15-\mathrm{H} 15 \mathrm{~A}$ & 0.9700 & $\mathrm{~N} 13-\mathrm{O} 19$ & $1.33(3)$ \\
\hline $\mathrm{C} 15-\mathrm{H} 15 \mathrm{~B}$ & 0.9700 & & \\
\hline $\mathrm{C} 2-\mathrm{C} 1-\mathrm{C} 6$ & $119.7(2)$ & $\mathrm{C} 17-\mathrm{C} 16-\mathrm{H} 16 \mathrm{~B}$ & 109.1 \\
\hline $\mathrm{C} 2-\mathrm{C} 1-\mathrm{N} 1$ & $121.2(2)$ & $\mathrm{H} 16 \mathrm{~A}-\mathrm{C} 16-\mathrm{H} 16 \mathrm{~B}$ & 107.8 \\
\hline $\mathrm{C} 6-\mathrm{C} 1-\mathrm{N} 1$ & $119.15(19)$ & $\mathrm{N} 2-\mathrm{C} 17-\mathrm{C} 16$ & $112.1(2)$ \\
\hline $\mathrm{C} 1-\mathrm{C} 2-\mathrm{C} 3$ & $120.4(3)$ & $\mathrm{N} 2-\mathrm{C} 17-\mathrm{H} 17 \mathrm{~A}$ & 109.2 \\
\hline $\mathrm{C} 1-\mathrm{C} 2-\mathrm{H} 2$ & 119.8 & $\mathrm{C} 16-\mathrm{C} 17-\mathrm{H} 17 \mathrm{~A}$ & 109.2 \\
\hline $\mathrm{C} 3-\mathrm{C} 2-\mathrm{H} 2$ & 119.8 & $\mathrm{~N} 2-\mathrm{C} 17-\mathrm{H} 17 \mathrm{~B}$ & 109.2 \\
\hline $\mathrm{C} 4-\mathrm{C} 3-\mathrm{C} 2$ & $119.8(3)$ & $\mathrm{C} 16-\mathrm{C} 17-\mathrm{H} 17 \mathrm{~B}$ & 109.2 \\
\hline $\mathrm{C} 4-\mathrm{C} 3-\mathrm{H} 3$ & 120.1 & $\mathrm{H} 17 \mathrm{~A}-\mathrm{C} 17-\mathrm{H} 17 \mathrm{~B}$ & 107.9 \\
\hline $\mathrm{C} 2-\mathrm{C} 3-\mathrm{H} 3$ & 120.1 & $\mathrm{~N} 2-\mathrm{C} 18-\mathrm{H} 18 \mathrm{~A}$ & 109.5 \\
\hline $\mathrm{C} 5-\mathrm{C} 4-\mathrm{C} 3$ & $120.1(3)$ & $\mathrm{N} 2-\mathrm{C} 18-\mathrm{H} 18 \mathrm{~B}$ & 109.5 \\
\hline $\mathrm{C} 5-\mathrm{C} 4-\mathrm{H} 4$ & 119.9 & $\mathrm{H} 18 \mathrm{~A}-\mathrm{C} 18-\mathrm{H} 18 \mathrm{~B}$ & 109.5 \\
\hline $\mathrm{C} 3-\mathrm{C} 4-\mathrm{H} 4$ & 119.9 & $\mathrm{~N} 2-\mathrm{C} 18-\mathrm{H} 18 \mathrm{C}$ & 109.5 \\
\hline $\mathrm{C} 4-\mathrm{C} 5-\mathrm{C} 6$ & $121.0(3)$ & $\mathrm{H} 18 \mathrm{~A}-\mathrm{C} 18-\mathrm{H} 18 \mathrm{C}$ & 109.5 \\
\hline $\mathrm{C} 4-\mathrm{C} 5-\mathrm{H} 5$ & 119.5 & $\mathrm{H} 18 \mathrm{~B}-\mathrm{C} 18-\mathrm{H} 18 \mathrm{C}$ & 109.5 \\
\hline $\mathrm{C} 6-\mathrm{C} 5-\mathrm{H} 5$ & 119.5 & $\mathrm{C} 15-\mathrm{N} 1-\mathrm{C} 1$ & $121.6(2)$ \\
\hline $\mathrm{C} 1-\mathrm{C} 6-\mathrm{C} 5$ & $119.0(2)$ & $\mathrm{C} 15-\mathrm{N} 1-\mathrm{C} 14$ & $120.1(2)$ \\
\hline $\mathrm{C} 1-\mathrm{C} 6-\mathrm{C} 7$ & $118.9(2)$ & $\mathrm{C} 1-\mathrm{N} 1-\mathrm{C} 14$ & $118.30(18)$ \\
\hline $\mathrm{C} 5-\mathrm{C} 6-\mathrm{C} 7$ & $122.1(2)$ & $\mathrm{C} 17-\mathrm{N} 2-\mathrm{C} 18$ & $112.6(2)$ \\
\hline $\mathrm{C} 6-\mathrm{C} 7-\mathrm{C} 8$ & $111.2(2)$ & $\mathrm{C} 17-\mathrm{N} 2-\mathrm{H} 1 \mathrm{~N}$ & 110.2 \\
\hline $\mathrm{C} 6-\mathrm{C} 7-\mathrm{H} 7 \mathrm{~A}$ & 109.4 & $\mathrm{C} 18-\mathrm{N} 2-\mathrm{H} 1 \mathrm{~N}$ & 109.9 \\
\hline $\mathrm{C} 8-\mathrm{C} 7-\mathrm{H} 7 \mathrm{~A}$ & 109.4 & $\mathrm{C} 17-\mathrm{N} 2-\mathrm{H} 2 \mathrm{~N}$ & 113.6 \\
\hline
\end{tabular}




\begin{tabular}{|c|c|c|c|}
\hline $\mathrm{C} 6-\mathrm{C} 7-\mathrm{H} 7 \mathrm{~B}$ & 109.4 & $\mathrm{C} 18-\mathrm{N} 2-\mathrm{H} 2 \mathrm{~N}$ & 109.1 \\
\hline $\mathrm{C} 8-\mathrm{C} 7-\mathrm{H} 7 \mathrm{~B}$ & 109.4 & $\mathrm{H} 1 \mathrm{~N}-\mathrm{N} 2-\mathrm{H} 2 \mathrm{~N}$ & 100.9 \\
\hline $\mathrm{H} 7 \mathrm{~A}-\mathrm{C} 7-\mathrm{H} 7 \mathrm{~B}$ & 108.0 & $\mathrm{O} 11-\mathrm{C} 21-\mathrm{C} 22$ & $126.3(2)$ \\
\hline $\mathrm{C} 9-\mathrm{C} 8-\mathrm{C} 7$ & $118.18(18)$ & $\mathrm{O} 11-\mathrm{C} 21-\mathrm{C} 26$ & $122.4(2)$ \\
\hline $\mathrm{C} 9-\mathrm{C} 8-\mathrm{H} 8 \mathrm{~A}$ & 107.8 & $\mathrm{C} 22-\mathrm{C} 21-\mathrm{C} 26$ & $111.3(2)$ \\
\hline $\mathrm{C} 7-\mathrm{C} 8-\mathrm{H} 8 \mathrm{~A}$ & 107.8 & $\mathrm{C} 23-\mathrm{C} 22-\mathrm{C} 21$ & $123.6(2)$ \\
\hline $\mathrm{C} 9-\mathrm{C} 8-\mathrm{H} 8 \mathrm{~B}$ & 107.8 & $\mathrm{C} 23-\mathrm{C} 22-\mathrm{N} 11$ & $116.1(2)$ \\
\hline $\mathrm{C} 7-\mathrm{C} 8-\mathrm{H} 8 \mathrm{~B}$ & 107.8 & $\mathrm{C} 21-\mathrm{C} 22-\mathrm{N} 11$ & $120.3(2)$ \\
\hline $\mathrm{H} 8 \mathrm{~A}-\mathrm{C} 8-\mathrm{H} 8 \mathrm{~B}$ & 107.1 & $\mathrm{C} 24-\mathrm{C} 23-\mathrm{C} 22$ & $119.8(2)$ \\
\hline $\mathrm{C} 10-\mathrm{C} 9-\mathrm{C} 14$ & $118.3(2)$ & $\mathrm{C} 24-\mathrm{C} 23-\mathrm{H} 23$ & 120.1 \\
\hline $\mathrm{C} 10-\mathrm{C} 9-\mathrm{C} 8$ & $115.8(2)$ & $\mathrm{C} 22-\mathrm{C} 23-\mathrm{H} 23$ & 120.1 \\
\hline $\mathrm{C} 14-\mathrm{C} 9-\mathrm{C} 8$ & $125.9(2)$ & $\mathrm{C} 23-\mathrm{C} 24-\mathrm{C} 25$ & $121.0(2)$ \\
\hline $\mathrm{C} 11-\mathrm{C} 10-\mathrm{C} 9$ & $122.6(3)$ & $\mathrm{C} 23-\mathrm{C} 24-\mathrm{N} 12$ & $119.7(3)$ \\
\hline $\mathrm{C} 11-\mathrm{C} 10-\mathrm{H} 10$ & 118.7 & $\mathrm{C} 25-\mathrm{C} 24-\mathrm{N} 12$ & $119.3(3)$ \\
\hline $\mathrm{C} 9-\mathrm{C} 10-\mathrm{H} 10$ & 118.7 & $\mathrm{C} 26-\mathrm{C} 25-\mathrm{C} 24$ & $118.7(2)$ \\
\hline $\mathrm{C} 10-\mathrm{C} 11-\mathrm{C} 12$ & $119.4(3)$ & $\mathrm{C} 26-\mathrm{C} 25-\mathrm{H} 25$ & 120.7 \\
\hline $\mathrm{C} 10-\mathrm{C} 11-\mathrm{H} 11$ & 120.3 & $\mathrm{C} 24-\mathrm{C} 25-\mathrm{H} 25$ & 120.7 \\
\hline $\mathrm{C} 12-\mathrm{C} 11-\mathrm{H} 11$ & 120.3 & $\mathrm{C} 25-\mathrm{C} 26-\mathrm{C} 21$ & $125.6(2)$ \\
\hline $\mathrm{C} 11-\mathrm{C} 12-\mathrm{C} 13$ & $119.6(3)$ & $\mathrm{C} 25-\mathrm{C} 26-\mathrm{N} 13$ & $116.1(2)$ \\
\hline $\mathrm{C} 11-\mathrm{C} 12-\mathrm{H} 12$ & 120.2 & $\mathrm{C} 21-\mathrm{C} 26-\mathrm{N} 13$ & $118.3(2)$ \\
\hline $\mathrm{C} 13-\mathrm{C} 12-\mathrm{H} 12$ & 120.2 & $\mathrm{O} 13-\mathrm{N} 11-\mathrm{O} 12$ & $121.8(3)$ \\
\hline $\mathrm{C} 14-\mathrm{C} 13-\mathrm{C} 12$ & $121.7(3)$ & $\mathrm{O} 13-\mathrm{N} 11-\mathrm{C} 22$ & $118.2(3)$ \\
\hline $\mathrm{C} 14-\mathrm{C} 13-\mathrm{H} 13$ & 119.2 & $\mathrm{O} 12-\mathrm{N} 11-\mathrm{C} 22$ & $120.0(2)$ \\
\hline $\mathrm{C} 12-\mathrm{C} 13-\mathrm{H} 13$ & 119.2 & $\mathrm{O} 14-\mathrm{N} 12-\mathrm{O} 15$ & $124.2(3)$ \\
\hline $\mathrm{C} 13-\mathrm{C} 14-\mathrm{C} 9$ & $118.4(2)$ & $\mathrm{O} 14-\mathrm{N} 12-\mathrm{C} 24$ & $118.2(3)$ \\
\hline $\mathrm{C} 13-\mathrm{C} 14-\mathrm{N} 1$ & $118.0(2)$ & $\mathrm{O} 15-\mathrm{N} 12-\mathrm{C} 24$ & $117.6(3)$ \\
\hline $\mathrm{C} 9-\mathrm{C} 14-\mathrm{N} 1$ & $123.6(2)$ & $\mathrm{O} 18-\mathrm{N} 13-\mathrm{O} 16$ & $76.0(10)$ \\
\hline $\mathrm{N} 1-\mathrm{C} 15-\mathrm{C} 16$ & $126.5(2)$ & $\mathrm{O} 18-\mathrm{N} 13-\mathrm{O} 17$ & $67.5(10)$ \\
\hline $\mathrm{N} 1-\mathrm{C} 15-\mathrm{H} 15 \mathrm{~A}$ & 105.7 & $\mathrm{O} 16-\mathrm{N} 13-\mathrm{O} 17$ & $121.7(3)$ \\
\hline $\mathrm{C} 16-\mathrm{C} 15-\mathrm{H} 15 \mathrm{~A}$ & 105.7 & $\mathrm{O} 18-\mathrm{N} 13-\mathrm{O} 19$ & $119.9(15)$ \\
\hline $\mathrm{N} 1-\mathrm{C} 15-\mathrm{H} 15 \mathrm{~B}$ & 105.7 & $\mathrm{O} 16-\mathrm{N} 13-\mathrm{O} 19$ & $82.9(11)$ \\
\hline $\mathrm{C} 16-\mathrm{C} 15-\mathrm{H} 15 \mathrm{~B}$ & 105.7 & $\mathrm{O} 17-\mathrm{N} 13-\mathrm{O} 19$ & $78.2(11)$ \\
\hline $\mathrm{H} 15 \mathrm{~A}-\mathrm{C} 15-\mathrm{H} 15 \mathrm{~B}$ & 106.1 & $\mathrm{O} 18-\mathrm{N} 13-\mathrm{C} 26$ & $127.0(10)$ \\
\hline $\mathrm{C} 15-\mathrm{C} 16-\mathrm{C} 17$ & $112.5(2)$ & $\mathrm{O} 16-\mathrm{N} 13-\mathrm{C} 26$ & $119.3(3)$ \\
\hline $\mathrm{C} 15-\mathrm{C} 16-\mathrm{H} 16 \mathrm{~A}$ & 109.1 & $\mathrm{O} 17-\mathrm{N} 13-\mathrm{C} 26$ & $118.9(3)$ \\
\hline $\mathrm{C} 17-\mathrm{C} 16-\mathrm{H} 16 \mathrm{~A}$ & 109.1 & $\mathrm{O} 19-\mathrm{N} 13-\mathrm{C} 26$ & $112.5(11)$ \\
\hline $\mathrm{C} 15-\mathrm{C} 16-\mathrm{H} 16 \mathrm{~B}$ & 109.1 & & \\
\hline $\mathrm{C} 6-\mathrm{C} 1-\mathrm{C} 2-\mathrm{C} 3$ & $-0.4(4)$ & $\mathrm{C} 9-\mathrm{C} 14-\mathrm{N} 1-\mathrm{C} 15$ & $-136.8(2)$ \\
\hline $\mathrm{N} 1-\mathrm{C} 1-\mathrm{C} 2-\mathrm{C} 3$ & $179.7(2)$ & $\mathrm{C} 13-\mathrm{C} 14-\mathrm{N} 1-\mathrm{C} 1$ & $-133.9(2)$ \\
\hline $\mathrm{C} 1-\mathrm{C} 2-\mathrm{C} 3-\mathrm{C} 4$ & $0.0(4)$ & $\mathrm{C} 9-\mathrm{C} 14-\mathrm{N} 1-\mathrm{C} 1$ & $45.6(3)$ \\
\hline $\mathrm{C} 2-\mathrm{C} 3-\mathrm{C} 4-\mathrm{C} 5$ & $0.0(4)$ & $\mathrm{C} 16-\mathrm{C} 17-\mathrm{N} 2-\mathrm{C} 18$ & $174.3(3)$ \\
\hline $\mathrm{C} 3-\mathrm{C} 4-\mathrm{C} 5-\mathrm{C} 6$ & $0.4(4)$ & $\mathrm{O} 11-\mathrm{C} 21-\mathrm{C} 22-\mathrm{C} 23$ & $178.2(2)$ \\
\hline $\mathrm{C} 2-\mathrm{C} 1-\mathrm{C} 6-\mathrm{C} 5$ & $0.8(3)$ & $\mathrm{C} 26-\mathrm{C} 21-\mathrm{C} 22-\mathrm{C} 23$ & $0.1(3)$ \\
\hline $\mathrm{N} 1-\mathrm{C} 1-\mathrm{C} 6-\mathrm{C} 5$ & $-179.3(2)$ & $\mathrm{O} 11-\mathrm{C} 21-\mathrm{C} 22-\mathrm{N} 11$ & $-3.4(3)$ \\
\hline $\mathrm{C} 2-\mathrm{C} 1-\mathrm{C} 6-\mathrm{C} 7$ & $-176.5(2)$ & $\mathrm{C} 26-\mathrm{C} 21-\mathrm{C} 22-\mathrm{N} 11$ & $178.48(19)$ \\
\hline $\mathrm{N} 1-\mathrm{C} 1-\mathrm{C} 6-\mathrm{C} 7$ & $3.4(3)$ & $\mathrm{C} 21-\mathrm{C} 22-\mathrm{C} 23-\mathrm{C} 24$ & $-0.1(3)$ \\
\hline $\mathrm{C} 4-\mathrm{C} 5-\mathrm{C} 6-\mathrm{C} 1$ & $-0.8(4)$ & $\mathrm{N} 11-\mathrm{C} 22-\mathrm{C} 23-\mathrm{C} 24$ & $-178.6(2)$ \\
\hline $\mathrm{C} 4-\mathrm{C} 5-\mathrm{C} 6-\mathrm{C} 7$ & $176.4(2)$ & $\mathrm{C} 22-\mathrm{C} 23-\mathrm{C} 24-\mathrm{C} 25$ & $-0.6(4)$ \\
\hline $\mathrm{C} 1-\mathrm{C} 6-\mathrm{C} 7-\mathrm{C} 8$ & $70.7(3)$ & $\mathrm{C} 22-\mathrm{C} 23-\mathrm{C} 24-\mathrm{N} 12$ & $178.5(2)$ \\
\hline
\end{tabular}




$\begin{array}{ll}\mathrm{C} 5-\mathrm{C} 6-\mathrm{C} 7-\mathrm{C} 8 & -106.4(3) \\ \mathrm{C} 6-\mathrm{C} 7-\mathrm{C} 8-\mathrm{C} 9 & -69.7(3) \\ \mathrm{C} 7-\mathrm{C} 8-\mathrm{C} 9-\mathrm{C} 10 & -165.8(2) \\ \mathrm{C} 7-\mathrm{C} 8-\mathrm{C} 9-\mathrm{C} 14 & 14.5(3) \\ \mathrm{C} 14-\mathrm{C} 9-\mathrm{C} 10-\mathrm{C} 11 & 0.4(4) \\ \mathrm{C} 8-\mathrm{C} 9-\mathrm{C} 10-\mathrm{C} 11 & -179.3(2) \\ \mathrm{C} 9-\mathrm{C} 10-\mathrm{C} 11-\mathrm{C} 12 & -0.6(5) \\ \mathrm{C} 10-\mathrm{C} 11-\mathrm{C} 12-\mathrm{C} 13 & 0.5(5) \\ \mathrm{C} 11-\mathrm{C} 12-\mathrm{C} 13-\mathrm{C} 14 & -0.3(4) \\ \mathrm{C} 12-\mathrm{C} 13-\mathrm{C} 14-\mathrm{C} 9 & 0.1(4) \\ \mathrm{C} 12-\mathrm{C} 13-\mathrm{C} 14-\mathrm{N} 1 & 179.7(2) \\ \mathrm{C} 10-\mathrm{C} 9-\mathrm{C} 14-\mathrm{C} 13 & -0.2(3) \\ \mathrm{C} 8-\mathrm{C} 9-\mathrm{C} 14-\mathrm{C} 13 & 179.6(2) \\ \mathrm{C} 10-\mathrm{C} 9-\mathrm{C} 14-\mathrm{N} 1 & -179.7(2) \\ \mathrm{C} 8-\mathrm{C} 9-\mathrm{C} 14-\mathrm{N} 1 & 0.0(3) \\ \mathrm{N} 1-\mathrm{C} 15-\mathrm{C} 16-\mathrm{C} 17 & -136.0(3) \\ \mathrm{C} 15-\mathrm{C} 16-\mathrm{C} 17-\mathrm{N} 2 & 64.2(3) \\ \mathrm{C} 16-\mathrm{C} 15-\mathrm{N} 1-\mathrm{C} 1 & -3.1(4) \\ \mathrm{C} 16-\mathrm{C} 15-\mathrm{N} 1-\mathrm{C} 14 & 179.5(2) \\ \mathrm{C} 2-\mathrm{C} 1-\mathrm{N} 1-\mathrm{C} 15 & -63.9(3) \\ \mathrm{C} 6-\mathrm{C} 1-\mathrm{N} 1-\mathrm{C} 15 & 116.2(2) \\ \mathrm{C} 2-\mathrm{C} 1-\mathrm{N} 1-\mathrm{C} 14 & 113.6(2) \\ \mathrm{C} 6-\mathrm{C} 1-\mathrm{N} 1-\mathrm{C} 14 & -66.3(3) \\ \mathrm{C} 13-\mathrm{C} 14-\mathrm{N} 1-\mathrm{C} 15 & 43.6(3) \\ & \end{array}$

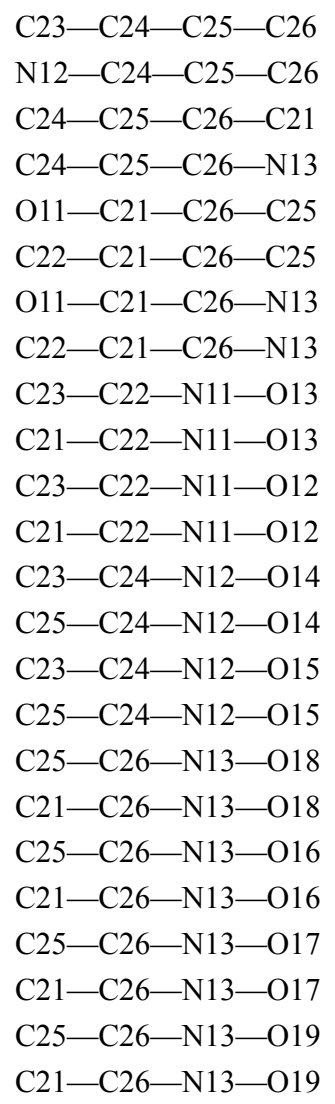

$1.3(4)$

$-177.8(2)$

$-1.4(4)$

$179.8(2)$

$-177.5(2)$

0.7 (3)

$1.3(3)$

$179.5(2)$

$-8.1(4)$

$173.4(3)$

$170.7(2)$

$-7.8(3)$

$-1.0(4)$

$178.1(3)$

$179.5(3)$

$-1.5(4)$

130.1 (13)

-48.8 (14)

$-135.5(3)$

45.6 (4)

47.6 (4)

$-131.3(3)$

-40.9 (12)

140.1 (12)

Hydrogen-bond geometry $\left(A,{ }^{\circ}\right)$

$$
\begin{aligned}
& D-\mathrm{H} \cdots A \\
& \mathrm{~N} 2-\mathrm{H} 1 \mathrm{~N} \cdots \mathrm{O} 11 \\
& \mathrm{~N} 2-\mathrm{H} 1 \mathrm{~N} \cdots \mathrm{O} 12 \\
& \mathrm{~N} 2-\mathrm{H} 2 \mathrm{~N} \cdots \mathrm{O} 21
\end{aligned}
$$

$D \multimap \mathrm{H}$
0.86
0.86
0.86

$D^{\cdots A} A$

2.817 (3)

3.001 (3)

$2.873(4)$
$D-\mathrm{H} \cdots A$

146

129

170 
Fig. 1

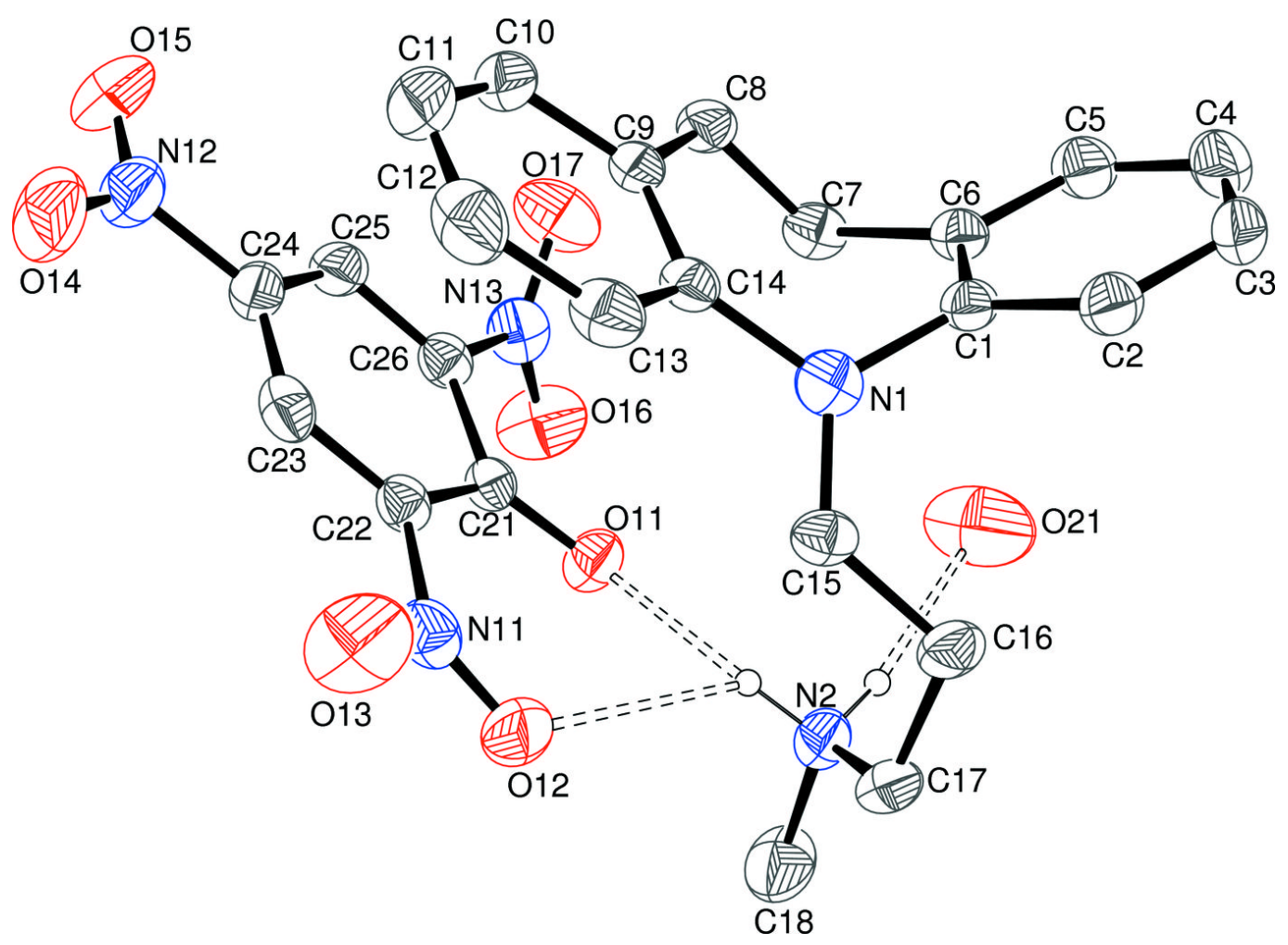

